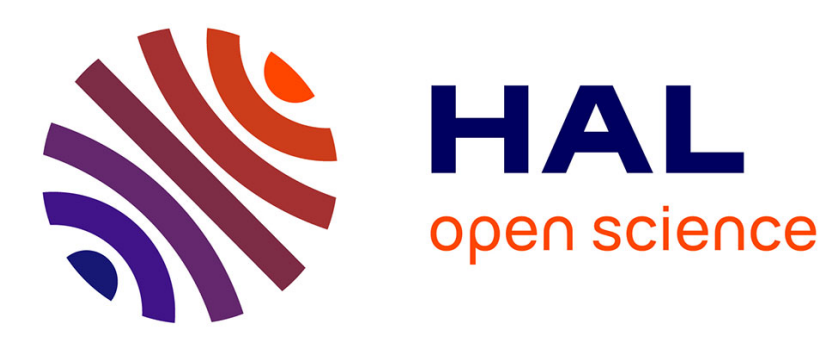

\title{
Caractérisation mécanique de micro-poutres par l'essai de flexion associé à une technique d'imagerie
}

\author{
A. Sergent, Laurent Robert, P. Delobelle, L. Bornier
}

\section{To cite this version:}

A. Sergent, Laurent Robert, P. Delobelle, L. Bornier. Caractérisation mécanique de micro-poutres par l'essai de flexion associé à une technique d'imagerie. Journal de Physique III, 1997, 7 (11), pp.21072129. 10.1051/jp3:1997244 . jpa-00249703

\section{HAL Id: jpa-00249703 https://hal.science/jpa-00249703}

Submitted on 1 Jan 1997

HAL is a multi-disciplinary open access archive for the deposit and dissemination of scientific research documents, whether they are published or not. The documents may come from teaching and research institutions in France or abroad, or from public or private research centers.
L'archive ouverte pluridisciplinaire HAL, est destinée au dépôt et à la diffusion de documents scientifiques de niveau recherche, publiés ou non, émanant des établissements d'enseignement et de recherche français ou étrangers, des laboratoires publics ou privés. 


\title{
Caractérisation mécanique de micro-poutres par l'essai de flexion associé à une technique d'imagerie
}

\author{
A. Sergent $\left({ }^{1}\right)$, L. Robert $\left({ }^{1,2}\right)$, P. Delobelle $\left({ }^{1, *}\right)$ et L. Bornier $\left({ }^{1}\right)$ \\ ( $\left.{ }^{1}\right)$ Laboratoire de Mécanique Applıquée R. Chaléat $\left({ }^{* *}\right)$, UFR Sciences et Techniques, \\ 24 rue de l'Épitaphe, 25030 Besançon Cedex, France \\ $\left({ }^{2}\right)$ Laboratorre de Physique et Métrologie des Oscillateurs, CNRS, 32 avenue de l'Observatorre, \\ 25030 Besançon Cedex, France
}

(Reçu le 18 décembre 1996, revisé le 24 juin 1997, accepté le 12 aout 1997)

PACS 62.20 Dc - Elasticity, elastic constants

PACS.62 20 Fe - Deformation and plasticity (including yield, ductility, and superplasticity)

PACS.68.60 Bs - Mechanical and acoustical properties

Résumé. - Durant cette dernière décennie le domame des micro-techniques a connu un formidable essor et maintenant le dimensionnement des micro-systèmes fait appel à de nombreuses disciplines scientifiques telles que l'électronique, les procédés d'obtention, l'optique, la mécanıque. . Celuı-ci s'effectue le plus souvent de manère globale et présente un caractère fortement interdisciplınaire Dans ce contexte, la connaissance des propriétés mécanıques des matérıux utilısés dans la conception des micro-systèmes, fortement dépendantes des procédés, est un aspect particulıèrement important. Dans cette logıque, de nouveaux moyens expérimentaux de caractérisation des matériaux en farbles dimensions ont été réalisés et, parmi les différentes possibilités, on présente l'essal de flexıon de micro-poutre encastrée-encastrée ou encastrée-libre. La mesure des déplacements globaux est réalısée simplement à l'aide d'une technıque d'ımagerıe optıque couplée à un traitement numérıque d'ımages Cette méthode a été validée sur des micropoutres de silıcium monocristallin et condunt à des mesures très reproductibles. Une application sur des micro-poutres en nickel électro-déposé et obtenues par technologie LIGA est également présentée. Les résultats expérimentaux sont en bon accord avec ceux issus des calculs analytıque et numérıque, ce qui valide l'ensemble de l'expérience.

\begin{abstract}
During the last decade, the domain of micro-techniques has had a formidable development. Today, the separate study of components is replaced by the design of micro-system assemblıng all these domains (electronic, optıc, data processing. .) Meanwhile, the knowledge of constituant materials mechanical properties (elastic and inelastic) remains vital Therefore, new means to characterize thin film materials are needed Among possible tests, the bending test of clamped-clamped or clamped-free microbeams has been holded. We present this technique and the significant role played by the magery method to measure the beam deflection To validate the principle and the possibilities of this bending test we present several results obtained with single crystals of silicon and with electro-deposited nickel. The good correlation obtaned between the experiments and the calculated profiles of the deformed beams allows to validate the whole of the experimental set-up.
\end{abstract}

$\left(^{*}\right)$ Auteur auquel doit être adressée la correspondance $\left.{ }^{* *}\right)$ URA CNRS 04

(C) Les Éditıons de Physıque 1997 


\section{Introduction}

La conception et la réalisation de mécanismes de dimensions submillimétriques voire micrométriques est un domaine en pleine expansion faisant appel à de nombreuses disciplines scientifiques $[1,2]$. Ainsi, d'une manière générale, on peut assimiler l'architecture mécanique des micro-systèmes à un assemblage de plaques, poutres ou membranes en liaison avec un substrat plus massif, soumises à des sollicitations mécaniques s'apparentant à des efforts du type traction, torsion, flexion ou pression. La connalssance des propriétés mécanıques (élastique et inélastique) des matériaux utilisés pour la réalisation des micro-systèmes représente un point essentiel si l'on veut quantifier par des méthodes numériques du type Éléments-Finis les fonctionnalités des architectures réalisées. De plus, ces propriétés dépendent fortement des processus d'obtention des matériaux [3,4], donc la compréhension des liens existant entre procédés, micro-structures et caractéristıques de la déformation constitue un axe de recherche important

L'ensemble de ces considérations ont conduit au développement de nouveaux moyens d'essais pour la caractérisation des matériaux en faibles dimensıons, par exemple ; l'essai de traction uniaxiale [5], l'essai de gonflement de membrane [6-8], l'essai de nano-indentation [9, 10] et l'essai de flexion [11-13].

C'est de ce dernier type d'essai, assez répandu dans le domaine des matériaux en faible épaisseur, et dans sa version flexion de poutre encastrée-encastrée et encastrée-libre, dont il sera question dans le présent artıcle. Cependant, alors que le principe de l'essai est assez simple, l'une des difficultés expérimentales réside dans la mesure quasi-instantanée et sans contact de la déformée de la poutre. Classiquement, on mesure la flèche en un point particulier, généralement au point d'application de la charge, ce qui peut conduire à des erreurs significatives si l'on ne connaît pas précisément d'une part la géométrie globale de la poutre, particulièrement au volsinage de l'encastrement, et d'autre part la position du point d'application de l'effort qui peut se déplacer lors du fléchissement. Notons également que l'interprétation théorique de la relation effort-flèche en tous points de la poutre, dans le cas des grands déplacements, doit être rigoureuse et nécessite une confrontation avec les calculs numériques.

Présentement, on réalise un système d'imagerie optique couplé à un traitement numérique des images enregistrées par une caméra C.C.D., ce qui permet de visualiser en temps réel l'état global de la déformée. Il s'agit donc d'une méthode de mesure dés déformées globales qui, connaissant l'effort appliqué sur l'échantillon, permet d'évaluer la relation effort-déformation intrinsèque au matériau testé. On valide la méthode sur des échantıllons mono-cristallins de silicium, matériau de référence dans le domane des micro-techniques, et quelques applications réalisées sur des micro-poutres en nickel électro-déposé et obtenues par micro-moulage sont également présentées.

\section{Présentation de l'essai}

2 1. PRINCIPE DE L'ESSAI. - Les épaisseurs visées des poutres à tester se situent à l'échelle des architectures des micro-systèmes à réalıser, donc typıquement de 10 à $200 \mu \mathrm{m}$, ce qui implique que la manipulation des échantillons est délicate et les forces mises en jeu lors du fléchissement assez faibles. Notons que pour des micro-poutres extrêmement minces, dont l'épaisseur est inférieure à $2 \mu \mathrm{m}$, les essais peuvent être réalısés à l'aide d'un appareillage extrêmement précis de nano-indentation $[14,15]$ mais dont les capacités en charges et déplacements sont limitées. L'expérience présentement développée se situe donc, vis-à-vis de la taille des poutres à tester, à une échelle supérieure à celle accessible au nano-indenteur, d'où la complémentarité des deux tests. Nous reviendrons ultérieurement sur ce point puisque des résultats issus de ces deux expériences seront comparés. De plus, toujours en ce qui concerne la forme des poutres, nous nous 
sommes orientés vers deux types de géométrie correspondant respectivement à la flexion quatre points de poutres Encastrée-Encastrée (E.E) et à la flexion simple de poutre Encastrée-Libre (E.L). Ces deux types de conditions aux limites impliquent bien évidemment des amplitudes de chargement et des distributions de contraintes dans la poutre assez différentes. Ainsi, dans les poutres E.E et entre les deux points d'application de la charge le moment de flexion est à peu près constant et l'effort tranchant quasiment nul, contrairement aux poutres E.L. où ces deux quantités sont conséquentes. Compte tenu des conditions d'encastrement à réaliser le plus proprement possible, les structures sont constituées d'un cadre rigide dans lequel sont usinés, au sens large du terme, les micro-poutres L'ensemble des éléments qui viennent d'être précisés nous ont fait choisir une longueur de poutre E.E. de $10 \mathrm{~mm}$ pour un entr'axe entre les points d'application des efforts de $5 \mathrm{~mm}$, et une longueur d'environ $5 \mathrm{~mm}$ pour les poutres E.L. En dernier lieu, précisons que le montage expérimental devra être capable de tester la réponse inélastique des poutres, c'est-à-dire être asservi en force et déplacement afin de réaliser des conditions de fluage, relaxation et cyclage à faible fréquence.

2.2. RÉAlisation MÉCANique DE L'ESSAi. - Un schéma synoptique de l'ensemble de l'expérience est présenté sur la figure la. Notons que l'ensemble du banc d'essai repose sur un marbre anti-vibratoire. La poutre, solidarre de son support rigide fixé sur une platine à l'arde de deux leviers, est fléchie par l'intermédiaire d'un simple ou double stylet (poutre E L. ou E.E.) monté sur un empilement de deux translateurs piezo-électriques. Cet ensemble est positionné sur un capteur de force, en l'occurrence une micro-balance, qui enregistre l'effort appliqué. Le positionnement précis des stylets par rapport à l'échantillon est réalisé par une table micrométrique trois axes. Compte tenu des dimensions des éprouvettes, seules des mesures sans contact permettent d'accéder aux déplacements, solt pour la réalisation de l'asservissement en position, soit pour l'obtention de la déformée complète. Pour ce qui concerne l'asservissement, la connaissance du déplacement des stylets est suffisante, ce qui est réalisé par un interféromètre laser visant un petit miroir solidaire de la platine supportant les stylets. Par contre, l'enregistrement de la déformée totale de la poutre, pouvant être considérée comme une mesure extensométrique locale, est assuré par un système d'imagerie comprenant : un anamorphoseur, une caméra C.C.D., une carte image et un ordinateur. Nous reviendrons dans le paragraphe suivant sur la description de ce système optique.

Une gestion informatique de tous les composants actifs de cette expérience assure :

- le pilotage et l'asservissement de la partie mécanique, à savoir, la réalisatıon .

. de rampes d'asservissement diverses en charge ou en déplacement,

- de cyclage à des fréquences pouvant aller jusqu'à $0,5 \mathrm{KHz}$,

. de relaxation ; asservissement en déplacement à l'aide de l'interféromètre laser,

. de fluage ; asservissement en charge avec la micro-balance,

- l'acquisition et le traitement des images, à savoir :

- le traitement deux dimensions de l'image,

. la visualisation trois dimensions à l'écran.

Notons que la synchronisation des deux ordinateurs assurant respectivement l'asservissement du système mécanique et la gestion des images permet de relier les mesures d'efforts aux mesures des déplacements selon l'axe $Z$ (Fig 1a) sur l'ensemble de la poutre. 


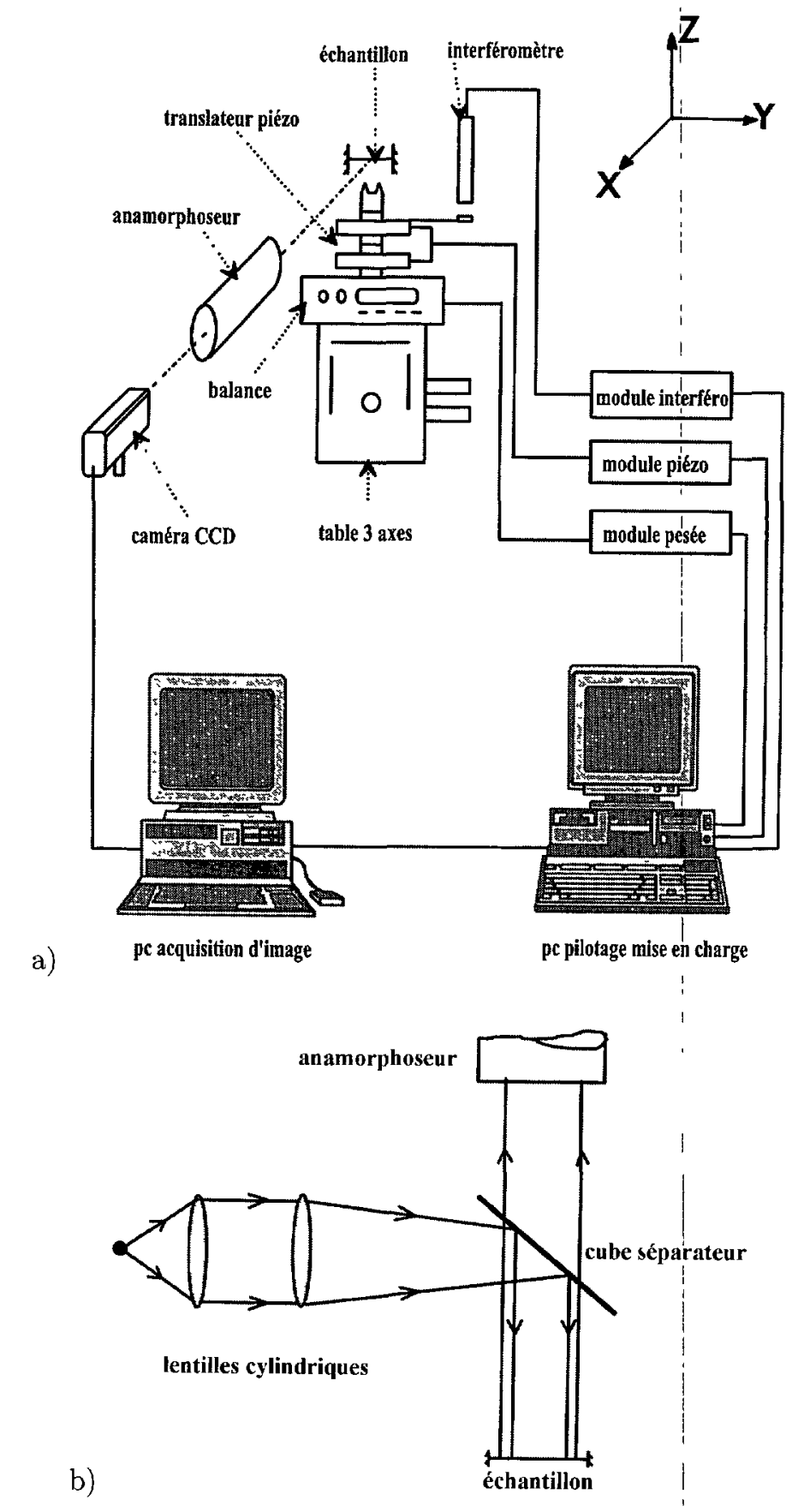

Fig. 1. - a) Schéma synoptıque du banc d'essais. b) Montage optıque pour éclairer l'échantillon. (a) Schematıc diagram depictıng the experimental setup for the bending test. b) Optical device to realize the lighting of the specimen.] 
2.3. Visualisation des ÉCHANTILlons ET DÉTERMination DES DÉPlaCEMENTS. - Rappelons qu'il s'agit d'une méthode de détermination de la déformée globale de l'échantillon et non d'une mesure ponctuelle Elle présente l'avantage d'avoir une vision d'ensemble de la géométrie de la structure déformée, ce qui peut être intéressant dans le cas de microstructures plus complexes, particulièrement aux points d'encastrement et d'articulation. Dans le cas d'une poutre $\mathrm{E} \mathrm{E}$. on peut évaluer la contraction lors de son fléchissement, du moins si celle-ci est conséquente.

Les dimensions des éprouvettes testées imposant un rapport longueur sur épalsseur supérieur à 100 , la visualisation des déformées en utılısant un système optique classique de grossissement ne convient pas. De ce fait, afin d'appréhender l'ensemble de la poutre, on utilise un anamorphoseur de rapport 20 correspondant au grossissement différentiel entre les deux directions optiques $Y, Z$ (Fig. 1a). La poutre étant préalablement éclairée, l'acquisition de l'image est réalisée par une caméra C.C.D. reliée à un micro-ordinateur interfacé par une carte image. Les informations sont ensuite traitées par ce dernier.

L'utilisation d'un tel montage impose cependant le respect d'un certain nombre de conditions optiques sur le positionnement des éléments et l'éclairage de l'échantillon En effet, l'objet à. visualiser, la source lumineuse et la caméra doivent être dans un même plan et de plus, l'axe optique de l'anamorphoseur doit être parallèle à l'axe suıvant lequel l'objet réfléchit les rayons. Ceci conduit à doter les différents composants du montage d'un certain nombre de degrés de liberté (table $X, Y$ sur les différents éléments). La surface des échantillons à observer étant soit diffusante, soit réfléchissante, il faut éclairer cette surface de deux manières dıfférentes. Dans le cas d'une surface réfléchissante la source utilisée est une lampe spectrale blanche et les rayons doivent arriver perpendiculairement à la surface de la poutre. En outre, le faisceau doit converger avec une ouverture double de celle de la première lentille sphérique de l'anamorphoseur Ces impératifs ont conduit à utiliser un montage simple tel que celui représenté sur la figure $1 \mathrm{~b}$. Pour une surface diffusante, on peut s'affranchir des conditions d'éclairage précédentes et illuminer l'objet avec une diode laser munie d'une optıque intégrée permettant une illumination régulière de la surface de l'échantillon. Cependant, des grains de speckle apparaissent sur les mages enregistrées et un traitement mathématique de convolutıon permettant d'uniformiser l'éclairement les rend exploitables

Le traltement des images enregistrées par la caméra et stockées sur le disque du microordinateur s'effectue à l'aide de l'algorithme présenté sur la figure 2 Il permet d'accéder aux déplacements en tous points de la poutre et de remonter aux composantes de la contrainte.

Un exemple d'innage d'une poutre E.E. défléchie est donné sur la figure 3. Notons enfin que le système dort être étalonné, ce qui est réalisé en mesurant très précisément l'épaisseur des poutres testées au microscope confocale.

2.4. RÉsumé de la PRATIque et des PeRformanCes de L'essai. - Concrètement, l'essai se déroule de la manière suivante . une poutre est fixée par l'intermédiaire de deux leviers ou collée sur un support rigide. Le ou les stylets sont positionnés précisément par rapport à l'échantillon grâce à la table trois axes, la poutre étant visualisée à l'écran de l'ordinateur. Une image de référence de l'objet non déformé est enregistrée. Les translateurs piezo-électriques sont activés faisant fléchir la poutre. L'interféromètre laser mesure en continu les déplacements des points d'application de la charge tandis que la micro-balance enregistre le chargement appliqué. Un micro-ordinateur gère l'ensemble du banc assurant les asservissements nécessaires (charges ou déplacements) et pilote un autre ordinateur qui assure l'acquisition des images pour les chargements souhaités. Le traitement et l'analyse des images sont ensuite réalisés grâce au logiciel développé, ce qui permet d’accéder aux déplacements et aux valeurs des composantes de la contrainte dans le cas d'un chargement élastique. 


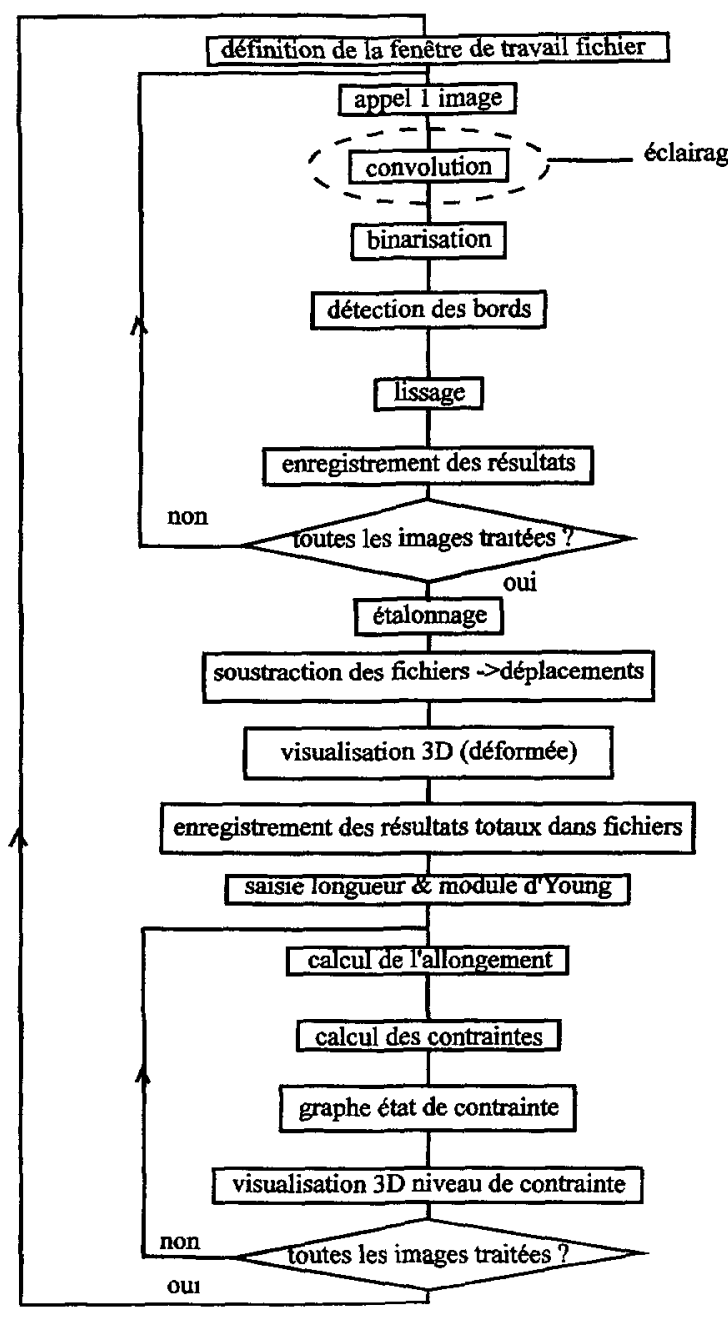

Fig 2. - Algorithme de traitement des images.

[Algorithm for the numerical treatment of the pictures ]

Les composants. les limites et les différentes sensibilités du banc d'essai se résument comme suit :

- mesure de charge : micro-balance Mettler PM $480 ; \pm 10^{-3}$ lg sur la plage $0-80 \mathrm{~g}$ et $\pm 10^{-2} \mathrm{~g}$ sur la plage $0-410 \mathrm{~g}$,

- déplacement maximal des stylets : $400 \mu \mathrm{m}$, deux translateurs piezo-électriques de $200 \pm 5 \times 10^{-4} \mu \mathrm{m}$,

- une table de positionnement $X, Y, Z$, à $1 \mu \mathrm{m}$ près,

- mesure des déplacements : interféromètre CSO-HC 250, $\pm 1 \mu \mathrm{m}$,

- un micro-ordinateur d'asservissement : PC 486, 


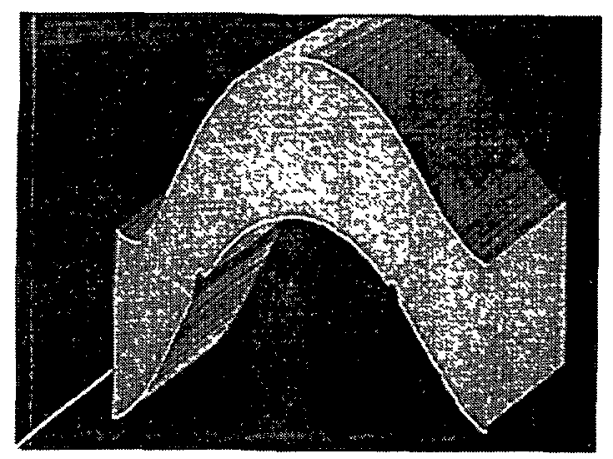

Poutre : $\mathrm{L}=10 \mathrm{~mm} ; \mathrm{l}=100 \mu \mathrm{m} ; \mathrm{h}=100 \mu \mathrm{m}$

Fig. 3. - Exemple d'mmage 3D de poutre E.E. déformée

[Example of a 3D visualisation of a clamped-clamped deflected beam.]

- un système d'éclairage laser ou lampe spectrale associés à des lentilles cylindrique et sphérique ainsi qu'à un cube séparateur,

- un anamorphoseur : rapport des grandissements de 20,

- une caméra C.C.D., IVC $500(512 \times 512$ pixels $)$; précision au pixel, soit $3 \mu \mathrm{m}$,

- un micro-ordinateur PC 486 assurant la saısie des images grâce à une carte image MATROX PIP 1024.

Compte tenu des différents agrandissements et de la caméra utilisée, on peut mesurer des flèches maximales de $300 \mu \mathrm{m}$ pour des poutres de $10 \mathrm{~mm}$ de long.

\section{Fabrication des échantillons}

3.1. CAS DU SILICIUM MONOCRISTALlin. - Le silicium monocristallin a été utilisé pour étalonner le banc de mesure. En effet, ses constantes élastiques sont parfaitement connues et son utilisation omni-présente dans les micro-dispositifs en font un matériau de référence en microtechnique. L'usinage des structures, effectué au sein de l'IMFC (Institut des Microtechniques de Franche-Comté), combine la photolithographie U.V. et la gravure chimique, c'est-à-dire l'attaque anisotrope du silicium.

Les différents types de poutres réalisées sont issues de wafers monocristallins double faces polies de $370 \mu \mathrm{m}$ d'épais et orientés selon les directıons $Z<100>$ et $Z<110>$. Nous n'entrerons pas dans les détails de la fabrication de ces structures, la figure 4 illustrant d'une façon synthétique les différentes étapes.

Cependant, compte tenu de l'attaque anisotrope du silicium par l'hydroxyde de potassium $(\mathrm{KOH})$, le cholx de l'un ou l'autre des deux types de wafer conditionne la géométrie de la section droite des poutres. En effet, l'utilisation de wafer $<110>$ permet d'obtenir des poutres de section droite rectangulaire, qui plus est de surfaces réfléchissantes, alors que l'utilisation de wafer $\langle 100\rangle$ conduit à des sections trapézoidales ayant des surfaces diffusantes et dont l'angle d'inclinaison des flancs est parfaitement connu. Les rugosités mesurées sont également différentes. 


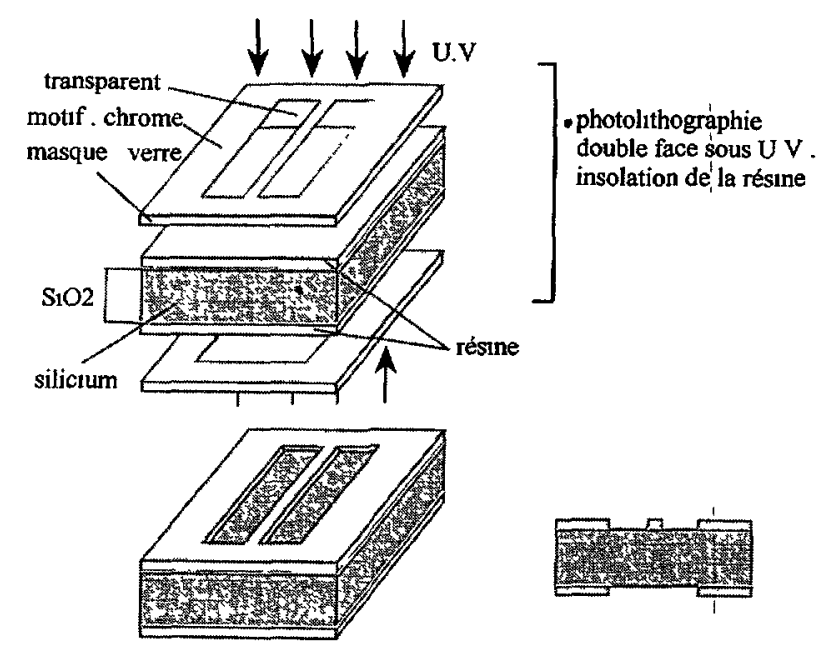

-révélation de la résıne et strippage · ouverture de la couche de $\mathrm{S}_{1} \mathrm{O} 2$ au $\mathrm{BHF}$

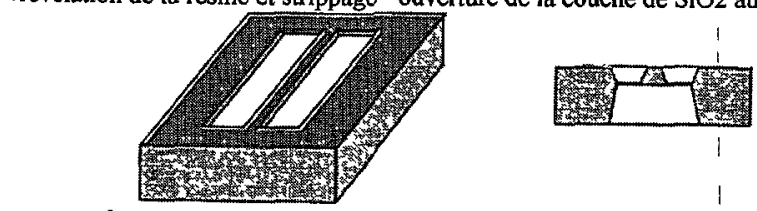

-attaque chimique au $\mathrm{KOH}$ la partie non protégée par le $\mathrm{S} 1 \mathrm{O} 2$ est attaquée
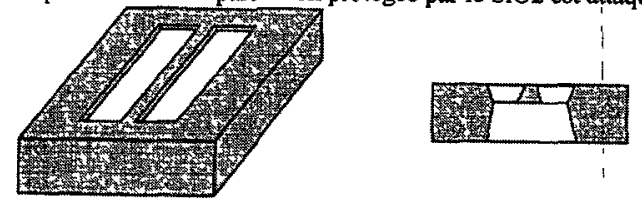

- forme finale après élımınatıon de l'oxyde de silıcium

Fig. 4 - Procédure d'obtention des poutres en silıcium

[Flowchart of the making of the silicon beams.]

La figure 5 représente d'une part la forme et l'orientation des sections droites des poutres et d'autre part, les géométries globales des structures réalisées : deux poutres $\mathrm{E} \mathrm{E}$ et quatre poutres E.L par échantillon Remarquons enfin qu'à l'aide de la plátıne de positionnement on peut tester les poutres selon deux directions perpendiculaires notées horizontale et verticale en référence au plan du wafer (Fig. 5). Les largeurs et les épaisseurs générées se situent entre 50 et $200 \mu \mathrm{m}$.

3.2. CAS DU NICKEL ÉLECTRO-DÉPosÉ. - Les potentialités du bánc d'essar sur un matériau dont les propriétés mécaniques sont a pror inconnues sont illustrées à l'arde de quelques résultats obtenus sur des poutres en nickel électro-déposé, réalisées par technologie LIGA. Ce matériau a ensuite été utilisé pour la réalisation de micro-pinces [16].

Les éprouvettes ont été élaborées à l'IMM (Institut des Microtechnıques de Mayence) par l'un des auteurs dans le cadre d'une collaboration avec l'IMFC En effet, cet institut a été l'initiateur de la technologie LIGA $[17,18]$ qui. rappelons-le signifie "Lithographie Galvanoformung, Abformung" Cette technique de micro-fabrication permet d'accéder à des micro-structures pseudo-tridimensionnelles à haut facteur de forme (rapport hauteur sur largeur pouvant 

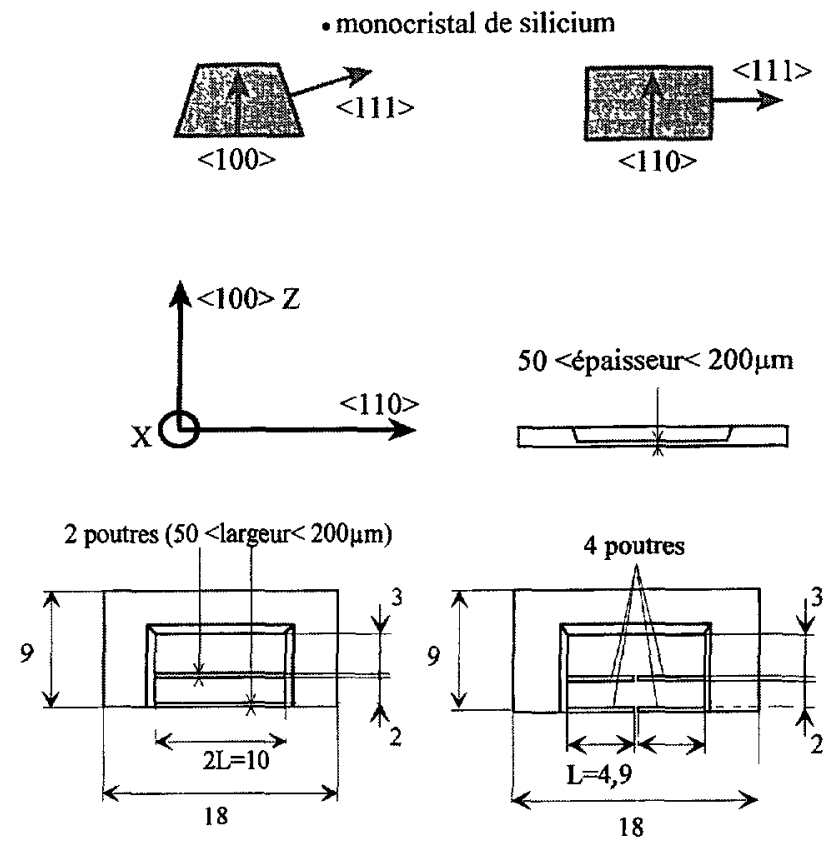

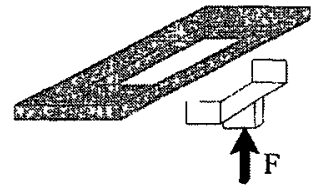

- poutre horizontale

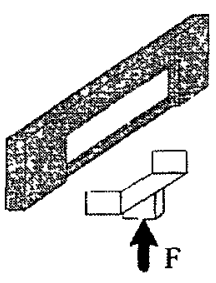

- poutre verticale

Fig 5 - Orientations et plans des poutres réalisées en slicium monocristallin.

[Orientations, dimensions and forms of the cross sections of the tested $\mathrm{S}_{1}$ specimens]

atteindre la centaine) de dımensions latérales pouvant atteindre le micron et ceci en utilisant une large variété de matérıaux (métaux, plastiques, céramiques ..).

Comme dans le cas précédent, nous n'entrerons pas dans les détails de la technologie d'élaboration de nos structures mais présentons, figure 6 , ses principales étapes.

Pour ce qui concerne la phase d'électro-formage des micro-poutres, le but étant d'étudier l'évolution des propriétés mécaniques du nickel électrolytique en fonction de la vitesse de déposition imposée par la densité de courant, les autres paramètres de déposition ont été fixés. Notons que la crosssance du nickel s'effectue sur une couche métallique sacrifiée en Cuivre $(30 \mu \mathrm{m})$. Le dépôt du métal est obtenu à partir d'une solution de sulfamate de nickel (100 $\mathrm{g}$ de $\left.\mathrm{N}_{\mathrm{l}} / 1\right)$, de chlorure de nickel $(5 \mathrm{~g} / \mathrm{l})$, d'acide borique $(40 \mathrm{~g} / \mathrm{l})$ et d'agent mouillant. La température du bain est de $50 \pm 0.5^{\circ} \mathrm{C}$ et le $\mathrm{PH}$ est contrôlé et fixé à 3,8 par ajout, sı nécessaire, d'acide sulfamique. Les densités de courant imposées sont de $0,25,0,5,1,0,2,6$ et $4 \mathrm{~A} \mathrm{dcm}^{-2}$ et pour chaque densité deux épaisseurs sont visées ; 50 et $200 \mu \mathrm{m}$ pour une largeur de $200 \mu \mathrm{m}$. L'étape d'ajustement de l'éparsseur s'effectue par polissage sımultané du nickel et du PMMA ce 


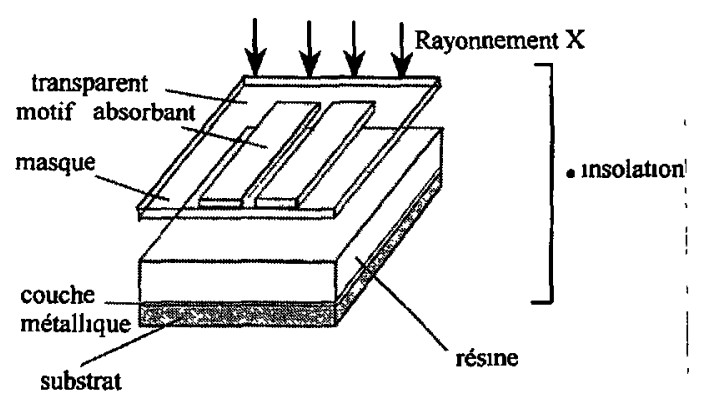

substrat

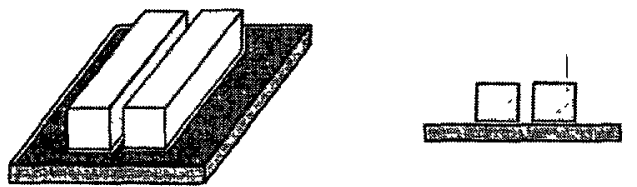

- révélation de la résine
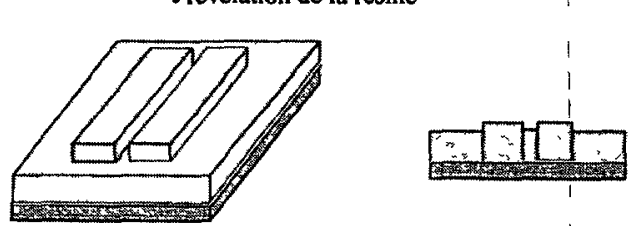

- électroformage
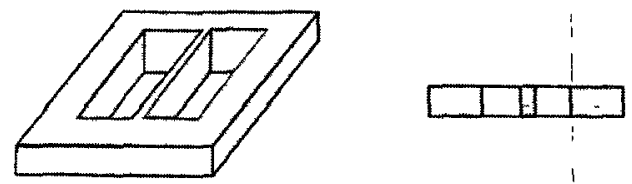

- forme finale après élımination de la couche métallı́que

Fig 6. - Procédure d'obtention des poutres en nickel électro-déposé.

[Flowchart of the making of the electro-deposited $\mathrm{Ni}$ beams.]

qui permet d'éviter l'arrachement des micro-structures. Un comparateur en contact permanent avec le substrat permet de stopper l'usinage lorsque la côte est atteinte.

\section{Confrontation calcul-expérience : validation de l'essai súr poutres silicium}

Deux types de calcul sont envisagés, soit analytique dans le cas de matériaux isotropes, pour des encastrements parfaits et des géométries de poutres sans défaut, soit numériques dans le cas contraire : matériaux anisotropes présentant des géométries complexes au nıveau des encastrements. Ces deux approches seront confrontées dans le cas présent.

4.1. MÉThodes analytiques. - Celles-ci sont abordées succinctement en annexe A pour les deux types de configuration de poutre [20]. Des relations flèche-effort exercé on peut déduire la valeur du module $E$ Pour un matériau monocristallin, connaissant ses constantes élastiques $C_{\imath \jmath}$ et son orientation par rapport aux axes géométriques de la poutre, , l est possible d'exprimer $E$ en fonction $\operatorname{des} C_{\imath \jmath}$ [21]

Pour les poutres encastrée-encastrées chargées en deux points symétriques il n'existe pas de solution analytique pure. Nous avons donc développé une méthode semi-analytique, l'ultime 
traitement des équations étant réalisé numériquement. Comme précédemment, on peut accéder au module d'Young du matériau mais en plus, compte tenu des conditions aux limites, aux contraintes résiduelles de tension dans la poutre (Annexe A).

Cependant, compte tenu des géométries des sections droites et des encastrements (géométrie complexe due à l'usinage anisotrope) il s'agit souvent d'une petite structure et une solution numérique du type éléments finis est beaucoup plus précise et adaptée.

4.2. MÉTHODES NUMÉRIQUES PAR ÉLÉMENTS FINIS. - Les deux codes utilisés sont ANSYS et Zebulon 7, respectivement pour les calculs élastique et élasto-plastique.

On travaille en élasticité anisotrope avec des élémentś volumiques, l'élément de base étant $3 \mathrm{D}$, quadratique, parallélépipédique à vingt noeuds. Le cristal est orienté par rapport au repère géométrique. Dans le maillage on tient compte de la géométrie exacte de la poutre (hormis sa rugosité), en particulier au niveau des différents plans de raccordement présents aux encastrements. Les calculs sont effectués en prenant pour les raideurs élastiques du silicium [21] : $C_{11}=165,7 \mathrm{GPa}, C_{12}=63,9 \mathrm{GPa}$ et $C_{44}=79,6 \mathrm{GPa}$.

4.3. RÉSUltats EXPÉRIMENTAUX ET VALIDATION DE L'EXPÉRIENCE - Comme mentionné précédemment, différentes orientations ont été testées correspondant d'une part à l'orientation initiale des wafers, $Z<100>$ et $Z<110>$, et d'autre part, au positionnement des échantillons par rapport à la direction d'application de la force, ces deux positions étant notées verticale et horizontale sur la figure 4. Bien que partant de wafers double faces polies, le processus d'attaque anisotrope crée dans le plan d'usinage une rugosité non négligeable des surfaces inférieures des poutres, différente selon l'orientation $Z$ et croissante avec le temps d'attaque, donc décroissance avec l'épaisseur de la poutre. Pour chacune des poutres étudiées les rugosités maximales $R_{\mathrm{tm}}$ ont été quantifiées par microscopie confocale. Par exemple, pour une épaisseur voisine de $100 \mu \mathrm{m}$, on reporte des valeurs maximales de l'ordre de $20 \mu \mathrm{m}$ pour $Z<110>$ et $3 \mu \mathrm{m}$ pour $Z<100>$. On ne peut donc pas négliger la rugosité (surtout pour $Z<110>$ ) et compte tenu de sa répartition spatiale homogène, on considérera que l'épaisseur moyenne de la poutre est donnée par [22] $e_{\mathrm{m}}=e_{\max }-\frac{R_{t_{m}}}{2}, e_{\max }$ étant l'épaisseur maximale mesurée par microscopie. De plus, les dimensions latérales des micro-structures pouvant présenter des fluctuations non répétitives à longue distance le long de la poutre, nous estimons les valeurs maximale et minimale de la largeur des poutres. Les calculs sont ensuite effectués avec ces deux valeurs extrêmes de manière à encadrer le comportement réel des éprouvettes. Notons enfin que la géométrie exacte des plans de raccordenuent au niveau de l'encastrement est prise en compte au niveau du maillage de la structure.

Ces précautions étant prises, on reporte sur les figures $7 a, b$, pour une même poutre E.E telle que $Z<100>$ présentant une rugosité assez faible, les résultats expérimentaux et théoriques des profils de déformées pour différentes valeurs du chargement et pour les deux positions horizontale et verticale. Pour les déformées, les deux courbes expérimentales correspondent à l'incertitude due au seuillage des niveaux de gris lors de la mesure du contour de la poutre, soit un pixel $( \pm 0.7 \mu \mathrm{m})$. De même, comme précısé précédemment, les deux résultats numériques obtenus par E F. tiennent compte des valeurs extrémales des dimensions, donc encadrent la solution de la poutre réelle. On note le bon accord entre expériences et calculs.

Sur les figures $8 \mathrm{a}, \mathrm{b}, \mathrm{c}, \mathrm{d}$, on reporte pour deux poutres issues de wafers orientés $Z<100>$ et $\langle 110\rangle$ et selon les deux positions horizontale et verticale, les relations expérimentales et théoriques de la flèche maximale au centre de la poutre en fonction du chargement. Les flèches maximales sont directement enregistrées par l'interféromètre et corroborent celles obtenues à partir du système optique. Ceci montre la bonne rigidité du montage et valide a posternor l'étalonnage du système optique. Les résultats obtenus par E.F. en prenant l'épaisseur moyenne 

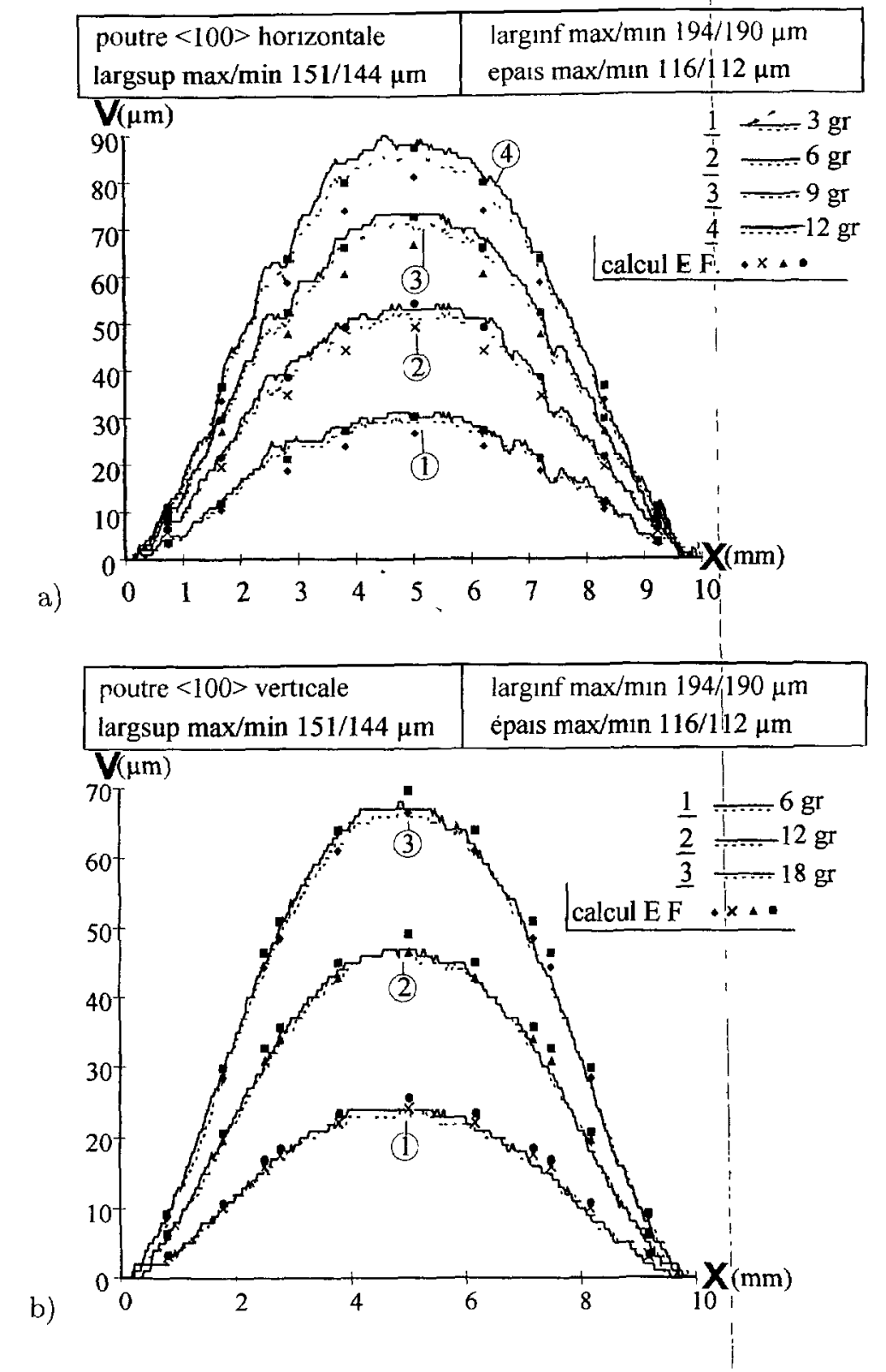

Fig 7. - Exemple de déformées de poutres E.E pour différentes valeurs dú chargement Confrontation calcul E.F et expérience a) poutre $<100>$ horizontale, b) poutre $<100\rangle$ verticale

[Example of the deformation of the clamped-clamped beam for different values of the loading. Comparıson between F.E calculation and experıment: a) horizontal $\langle 100\rangle$ beam, b) vertıcal $<100\rangle$ beam.] 

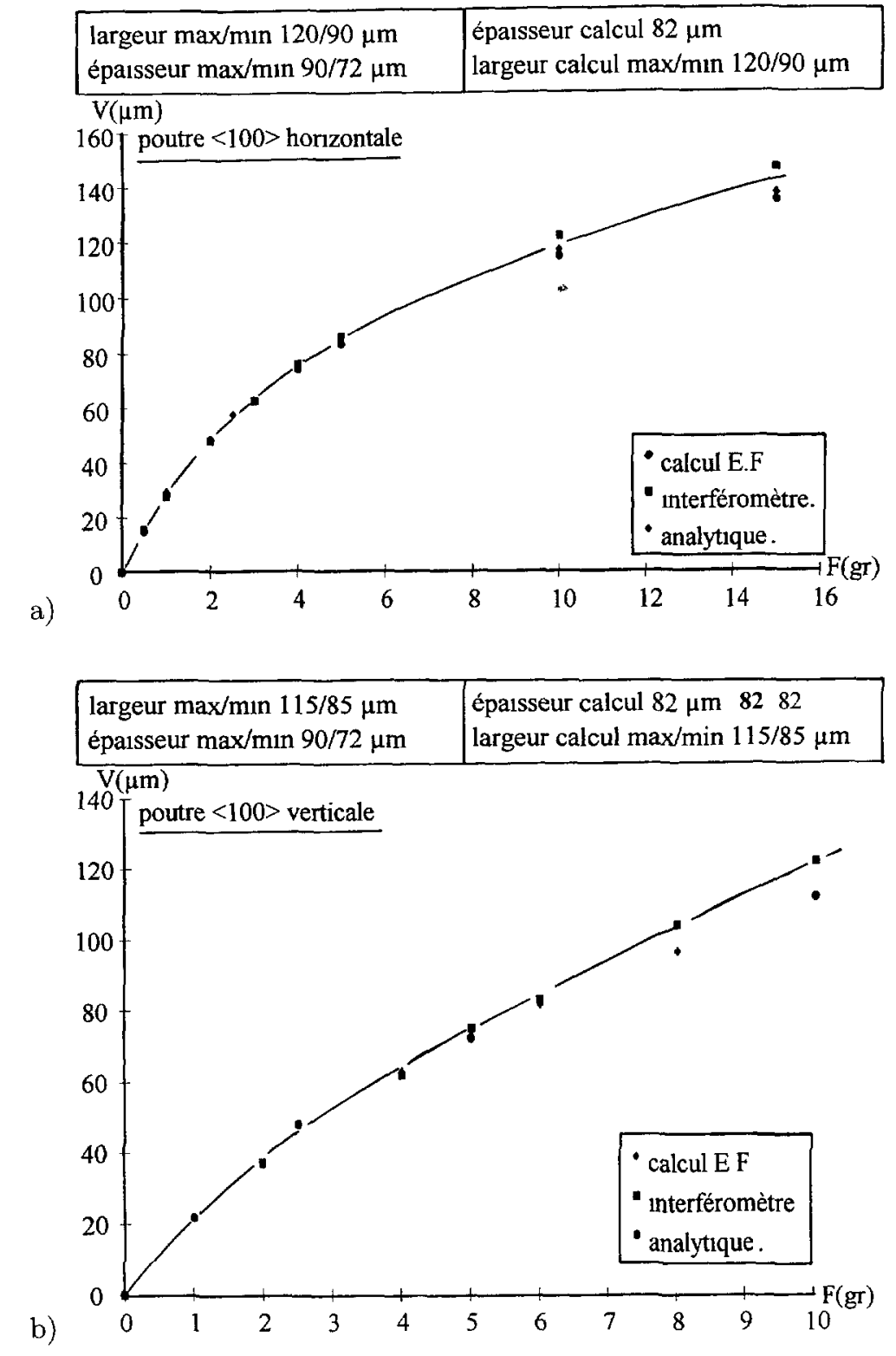

Fig 8. - Relation déflection maximale en fonction du chargement, $V=f(F)$. Confrontation calculexpérience a) poutre $\langle 100\rangle$ horizontale. b) poutre $\langle 100\rangle$ vertıcale. c) poutre $\langle 110\rangle$ horizontale, d) poutre $\langle 110\rangle$ verticale.

[Relationship between the maximum deflection of the clamped-clamped beam and the loading force. $V=f(F)$ Comparison calculation-experiment. a) horizontal beam $\langle 100\rangle$, b) vertical beam $\langle 100\rangle$, c) horizontal beam $\langle 110\rangle$, d) vertical beam $<110\rangle$.] 


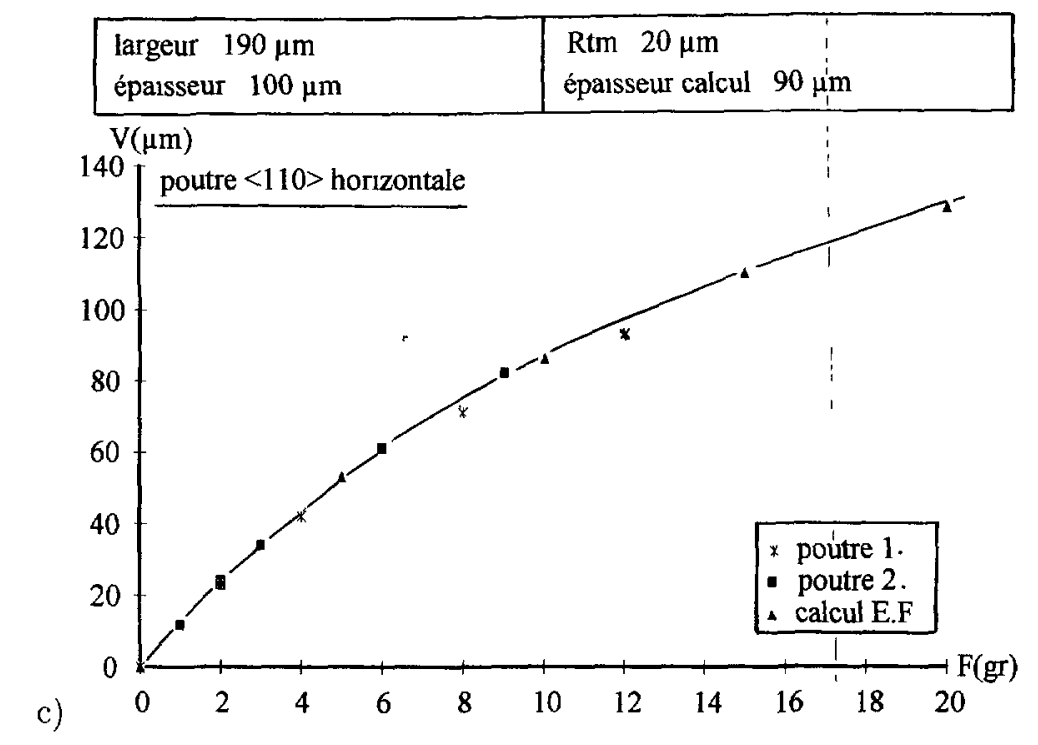

\begin{tabular}{|l|l|}
\hline \begin{tabular}{|l|} 
largeur $100 \mu \mathrm{m}$ \\
épaisseur $190 \mu \mathrm{m}$
\end{tabular} & $\begin{array}{l}\text { largeur calcul } 100 \mu \mathrm{m} \\
\text { éparsseur calcul } 190 \mu \mathrm{m}\end{array}$ \\
\hline
\end{tabular}

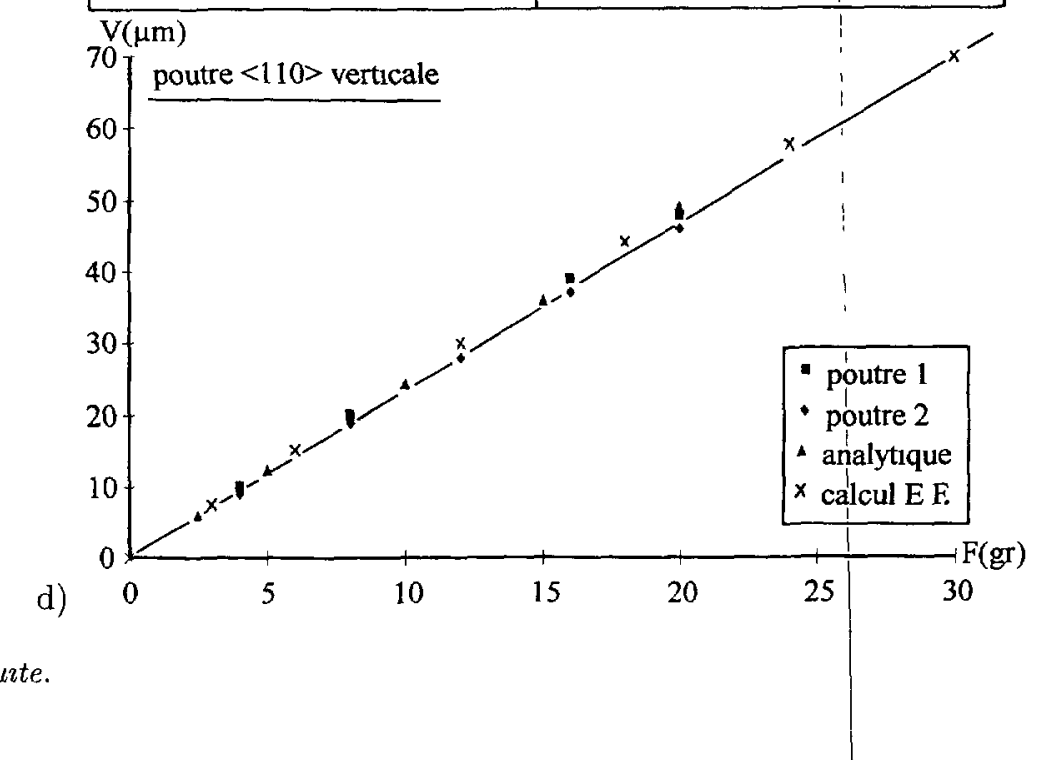

Fig. 8 - Surte.

[Continued.]

de la poutre sont en bon accord avec les résultats expérimentaux. De même, les calculs semıanalytiques, en considérant $e_{\text {moy }}$, les moments quadratiques adéquats (sections rectangulaire ou trapézoïdale) et en prenant les valeurs théoriques de $E$ issues de celles des $C_{\imath \jmath}$ et de l'orientation du monocristal, à savoir :

$$
\begin{aligned}
& Z<100>, X<110>, E_{\mathrm{x}}=\frac{4 C_{44}\left(C_{11}-C_{12}\right)\left(C_{11}+2 C_{12}\right)}{2 C_{11} C_{44}+\left(C_{11}-C_{12}\right)\left(C_{11}+2 C_{12}\right)}=168,9 \mathrm{GPa} \\
& Z<110>, X<\overline{1} 11>, E_{\mathrm{x}}=\frac{3 C_{44}\left(C_{11}+2 C_{12}\right)}{C_{44}+2 C_{11}+2 C_{12}}=187,5 \mathrm{GPa}
\end{aligned}
$$




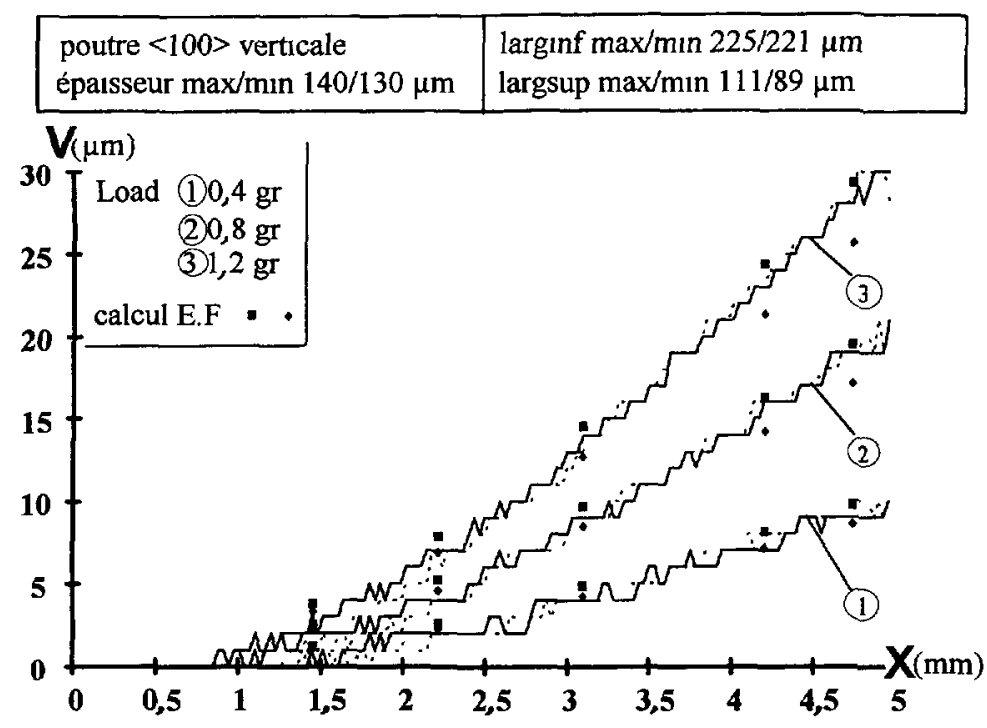

Fig. 9 - Exemple de déformées de poutre E.L. pour différentes valeurs du chargement Poutre $<110>$ verticale - Confrontation calcul-expérience.

[Example of the deformation of the free-clamped beam for different values of the loading: $<100>$ vertical beam - Comparison $\mathrm{F}$ E calculation-experiment.]

conduisent à des résultats proches de ceux calculés par E.F On montre ainsi que les défauts géométriques aux encastrements, compte tenu de la longueur de la poutre, n'ont qu'une faible influence sur l'amplitude de la déformée.

Des exemples de déformées réalisées sur une poutre E.L. orientée selon $Z<100>$ et verticale sont présentés sur la figure 9, ainsi que les prévisions issues du calcul E.F Là encore l'adéquation expérience-calcul est correcte. À noter qu'une campagne d'essais assez conséquente a été réalisée sur différentes géométries de poutres et que les résultats présentés n'en constitue qu'une très petite partie [23].

En conclusion, la bonne adéquation entre les déformées expérimentales et celles issues des calculs sur des poutres de géométries connues et réalisées dans un matériau dont les propriétés élastiques sont parfaitement établies, conduit à la validation finale de la technique expérimentale de l'essaỉ à tous les niveaux, à savoir - mécanique, optique. informatique ainsi qu'à la calibration des différents composants.

4.4. Potentialités de l'ESSAI SUR UN MATÉRIAU À CARACTÉRISTIQUES MÉCANIQUES INCONNUES. CAS DU NICKEL ÉLECTRODÉPOSÉ. - Comme nous l'avons évoqué précédemment, le but de l'étude [19] est la détermination des propriétés mécaniques du nickel électro-formé en fonction de la densité du courant d'électro-déposition, en vue de l'optimisation de la réalisation de micro-préhenseur [16]. Les poutres obtenues par technologie LIGA sont de très bonnes qualités, les tolérances sur les côtes étant inférieures à $0,5 \mu \mathrm{m}$. Le module d'Young déterminé analytiquement par la flexion élastique des poutres E.L. semble indépendant de la valeur de la densité de courant $J$, soit $E=175 \pm 5 \mathrm{GPa}$. À noter que les flèches mesurées en bout de poutre $\left(l \times e \cong 200 \times 200 \mu \mathrm{m}^{2}\right.$ et $\left.L=4,5 \mathrm{~mm}\right)$ sont de l'ordre d'une centaine de microns pour quelques grammes de chargement $(F \cong 5 \mathrm{~g})$. Quelques essais de flexion de poutres E.L. 


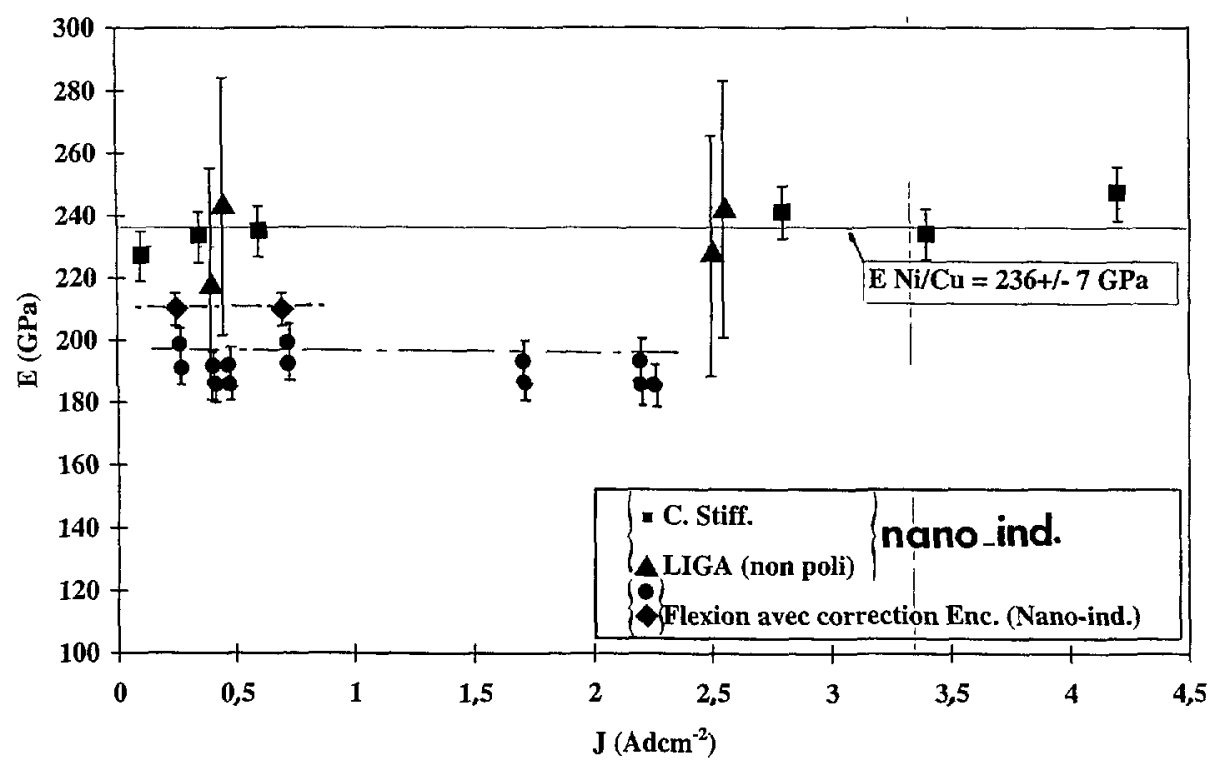

Fig. 10. - Poutres E L en nickel électro-déposé. Évolution du module d'Young avec la densité de courant $E=f(J)$. Confrontation avec les résultats obtenus en nano-mdentation et en micro-flexion.

[Free-clamped electro-deposited $N_{1}$ beam Evolution of the Young modulus as a function of the current density, $E=f(J)$. Comparison with the results obtained by nano-indentation and micro-flexion tests.]

effectués sur nano-indenteur conduisent à des résultats voisins, $E=184 \pm 3 \mathrm{GPa}$, d'où la complémentarité des deux types d'essais Cependant. dans ce dernier cas les forces et les déplacements mis en jeu sont très inférieurs à ceux du présent montage ; par exemple, pour une poutre telle que $l \times e=200 \times 192 \mu \mathrm{m}^{2}$ et $L=4.5 \mathrm{~mm}$ on enregistre un déplacement de $14,0 \mu \mathrm{m}$ pour un gramme appliqué. Si l'on tient compte par la méthode des E.F. de l'encastrement réel, le cadre des poutres est collé par une face sur le porte-échantillon, on calcule un module un peu plus élevé soit : $E=195 \mathrm{GPa}$ et $E=205 \pm 5 \mathrm{GPa}$ respectivement pour les deux méthodes (Fig. 10). Ces valeurs sont très proches de celle mentıonnée pour le nickel volumique $(E=210 \mathrm{GPa})$.

Des essais de nano-dureté effectués sur le support rigide des poutres conduisent d'une part lors de la phase d'indentation, à l'évolution de la dureté $H_{\mathrm{B}}$ en fonction de $J$ (Fig. 11 où l'on reporte également la micro-dureté Vickers $H_{\mathrm{v}}$ ) et d'autre part, lors du déchargement, à la valeur du module d'Young $[9,10]$. Les valeurs de $E$ déterminées par cette méthode sont reportées sur la figure 10 et sont indépendantes de $J$ comme pour les essais de flexion. Par contre la valeur moyenne est plus élevée ; $E=235 \pm 7 \mathrm{GPa}$. Ce constat déjà mentionné dans la littérature reste à expliquer.

Des essais de flexion quatre points sur éprouvettes E.E. de sections voisines de celles des poutres E.L. conduisent à des valeurs de module plus faibles, typiquement de l'ordre de 130 à $150 \mathrm{MPa}$. Cette observation ne peut s'expliquer que par l'intervention d'une contrainte résiduelle de compression résultant de la dilatation différentielle entre lè cuivre et le nickel lors du retour à la température ambiante après électro-dépositıon. Le calcul réalisé avec $\Delta T=35^{\circ} \mathrm{C}$ et les coefficients de dılatation des deux métaux considérés conduit à une contrainte thermique de compression de $\sigma_{0}^{\text {th }}=-20 \mathrm{MPa}$ (F1g. 12). Pour l'ensemble des échantillons E.E., connaissant la 


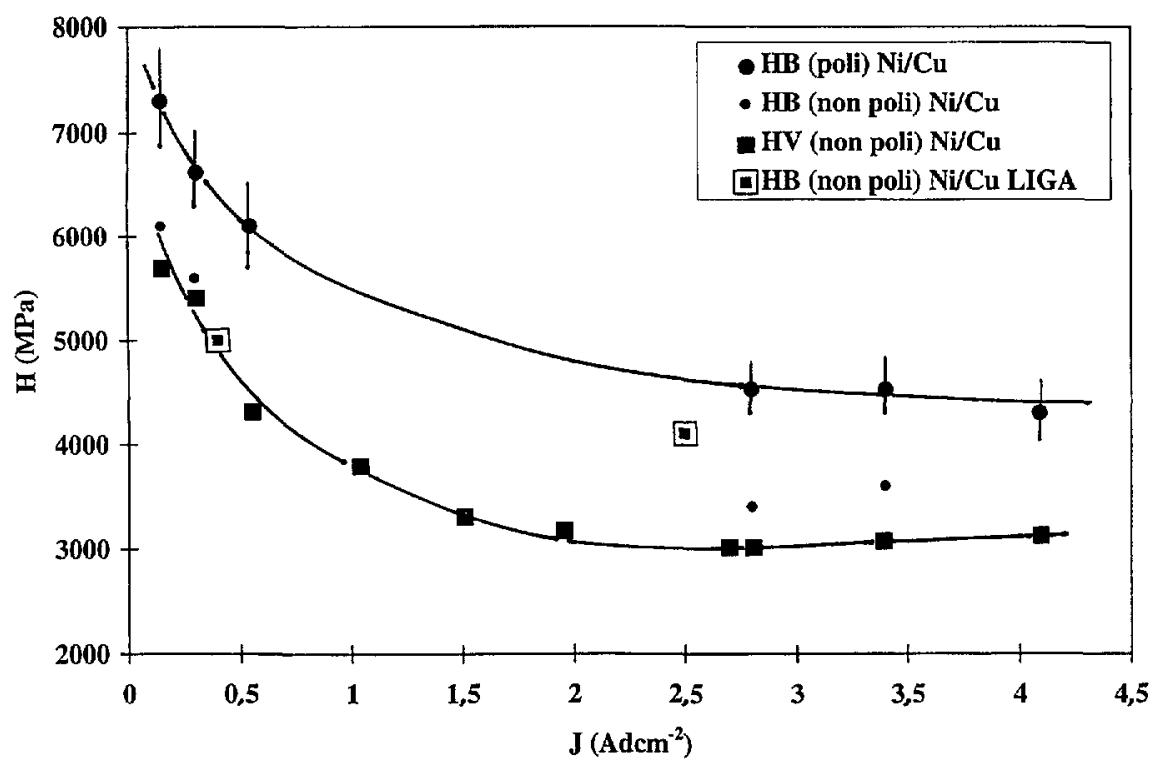

Fig 11 - Évolution de la dureté avec la densité de courant. $H=f(J)$.

[Evolution of the hardness with the current density $H=f(J)$.]

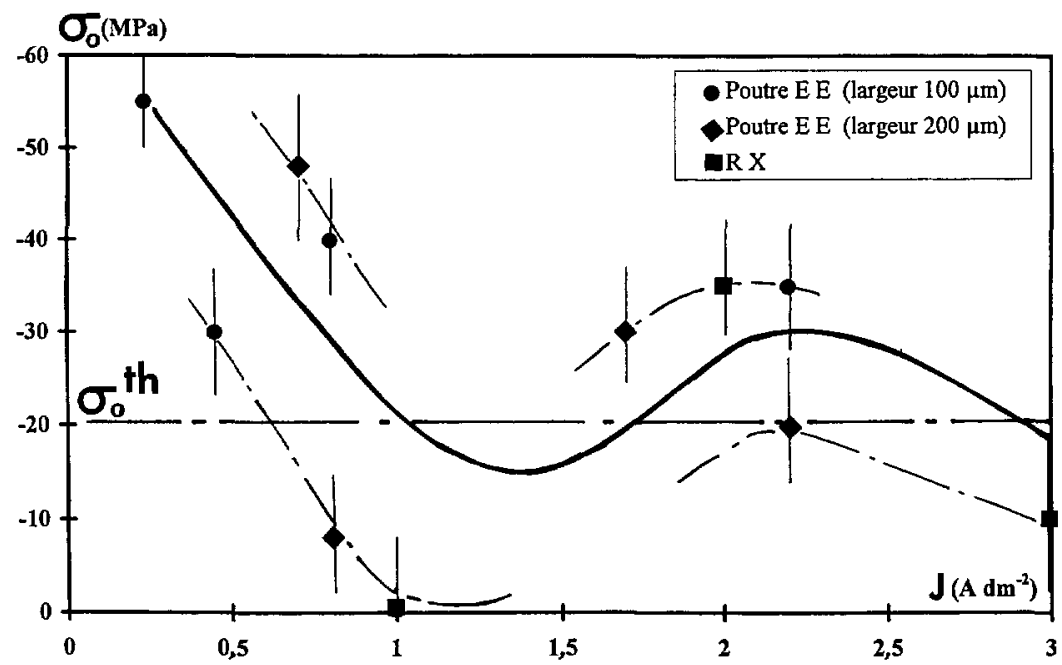

Fig. 12. - Évolution de la contrainte résiduelle avec la densité de courant, $\sigma_{0}=f(J)$.

[Evolution of the residual stress (clamped-clamped beams) with the current density: $\sigma_{0}=f(J)$.]

valeur du module, $E=200 \mathrm{GPa}$, on détermine $S_{o}$ (Annexe A) donc la contrainte de compression $\sigma_{0}$ de façon à obtenır la bonne déformée. Les valeurs calculées sont reportées figure 12 et évoluent entre 0 et $-60 \mathrm{MPa}$, donc de l'ordre de grandeur de la contrainte extrınsèque d'orıgıne thermique. Les fluctuations observées en fonction du paramètre $J$, si elles se confirment, ne 
a)

\begin{tabular}{|l|l|l|}
\hline $\begin{array}{l}\text { longueur } 45 \mathrm{~mm} \\
\mathrm{~J}=1,62 \mathrm{Adcm}-2\end{array}$ & $\begin{array}{l}\text { éparsseur } 167 \mu \mathrm{m} \\
\text { largeur } 200 \mu \mathrm{m}\end{array}$ \\
\hline
\end{tabular}

$\mathrm{F}(10 \mathrm{mN})$

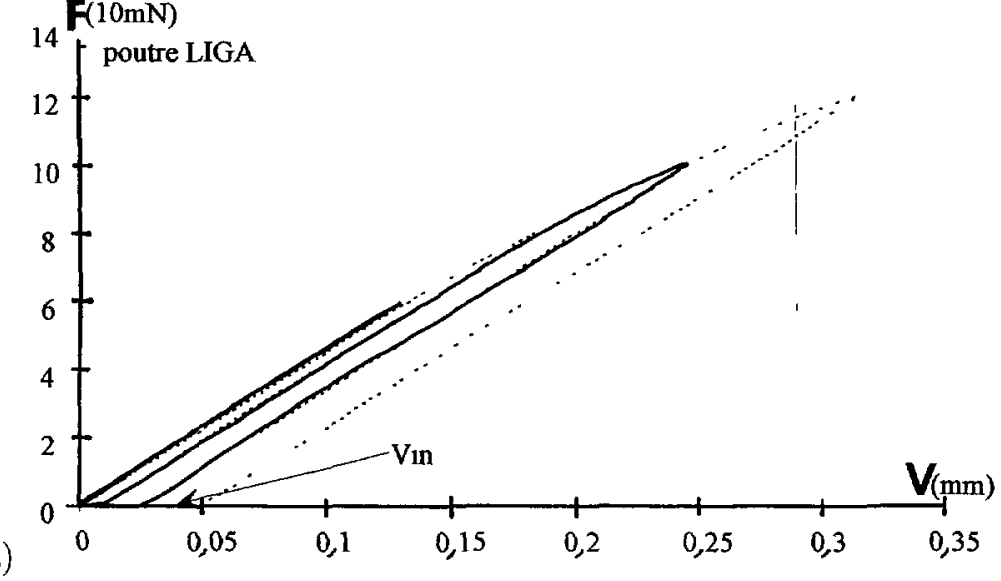

\begin{tabular}{|c|c|c|}
\hline $\begin{array}{l}\text { (1) } \mathrm{J}=0,25 \mathrm{Adcm}-2 \\
\mathrm{w}^{*} \mathrm{t}=200^{*} 204 \mu \mathrm{m} \\
\mathrm{L}=4.5 \mathrm{~mm}\end{array}$ & $\begin{array}{l}\text { (2) } \mathrm{J}=0,69 \text { Adcm-2 } \\
\mathrm{w}^{*} \mathrm{t}=200^{*} 192 \mu \mathrm{m} \\
\mathrm{L}=4,5 \mathrm{~mm}\end{array}$ & $\begin{array}{l}\text { (3) } \mathrm{J}=1,62 \text { Adcm-2 } \\
w^{*} \mathrm{t}=200^{*} 167 \mu \mathrm{m} \\
\mathrm{L}=4,5 \mathrm{~mm}\end{array}$ \\
\hline
\end{tabular}

b)

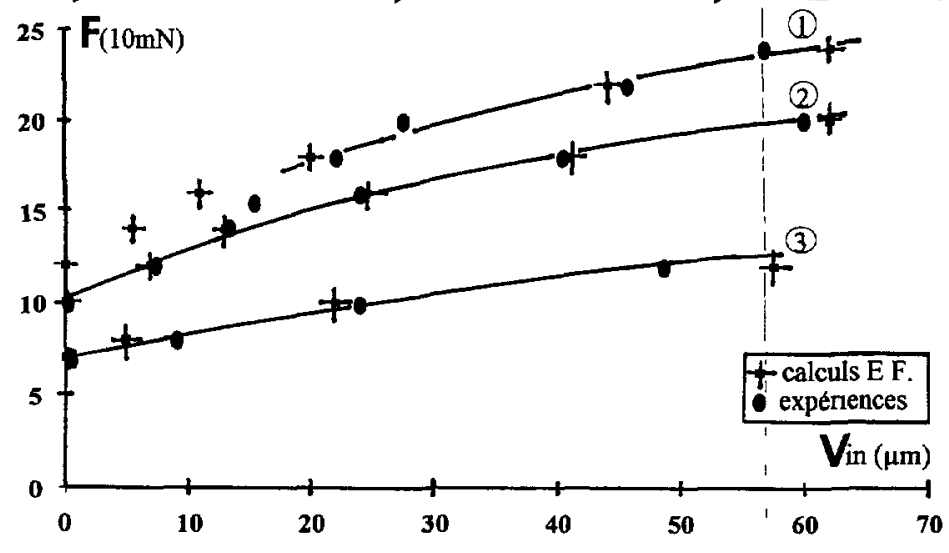

Fig. 13. - Comportement inélastique du nickel a a) Exemple de réponse inélastique de poutre E.L b) Évolution de la déformation inélastique avec la charge, $V_{1 \mathrm{n}}=f(F)$ pour tros densités de courant c) Modélisatıon E.F. du chargement correspondant à la figure 13a. d) Évolution des courbes d'écrouissage avec la densité de courant, $\sigma=f\left(\varepsilon^{\mathrm{T}}\right)$

[Inelastic behavior of the electro-deposited nickel a) Example of the inelastic response of a freeclamped beam, b) Evolution of the inelastic deformation with the loadıng, $V_{1 \mathrm{n}}=f(F)$, for three current densities, c) Numerical simulation of the response corresponding to the Figure 13a, d) Evolution of the strain hardening curves with respect to the current density, $\sigma=f\left(\varepsilon^{\mathrm{T}}\right)$.]

peuvent être que d'origine intrinsèque c'est-à-dire liées au phénomène de croissance du nickel sur le substrat. Quelques mesures de contraintes résiduelles par diffraction des R.X. sur les plans $<420>$ et en utilisant la méthode du $\sin ^{2} \psi[24]$ semblent confirmer ce type de variation (Fig. 12).

Si l'on s'intéresse aux propriétés inélastiques, on montre figure 11 que la dureté $H_{\mathrm{B}}$, liée 

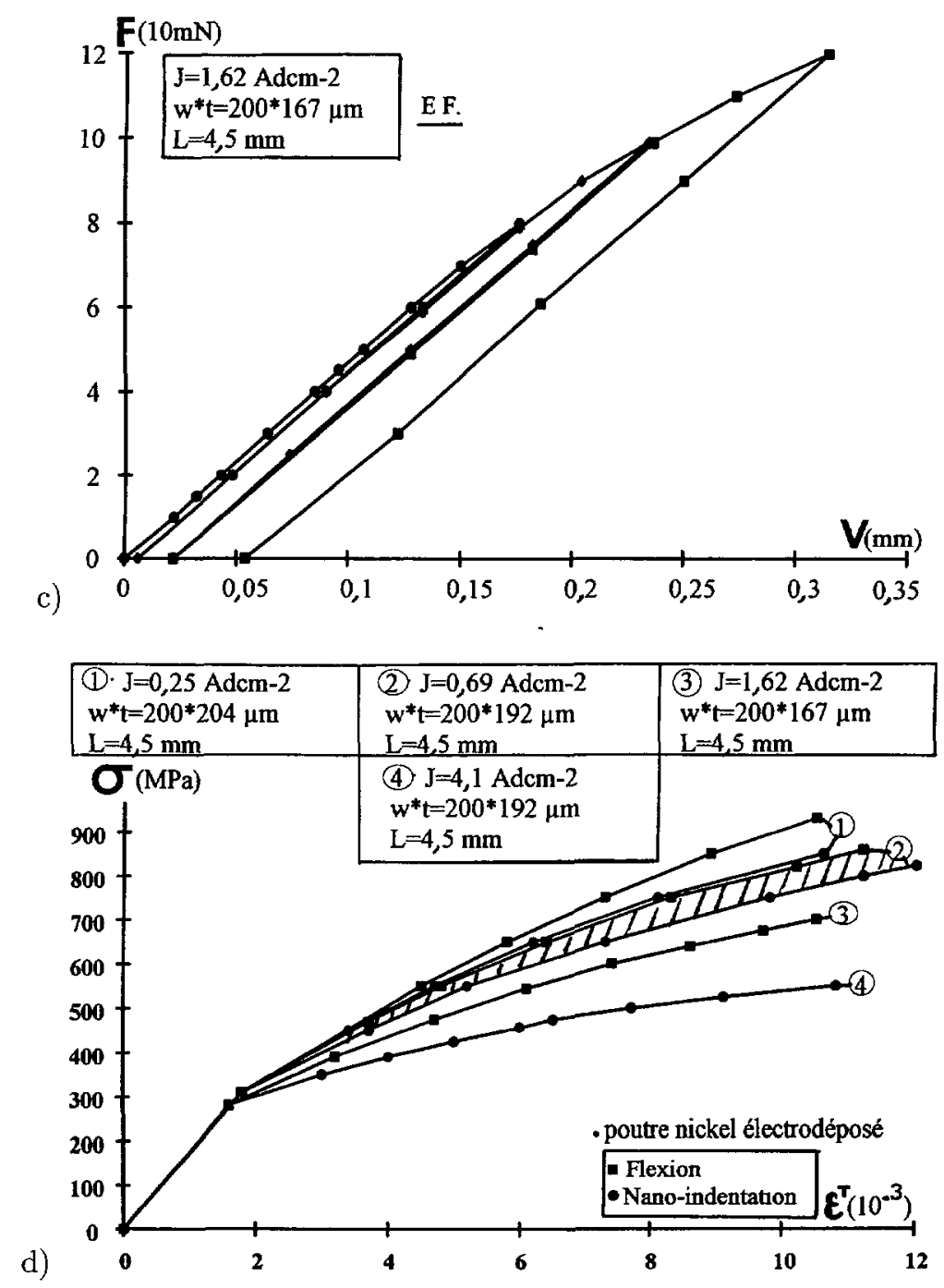

Fig. 13. - Sunte.

[Continued]

aux potentialités d'écrouissage du matériau, décroît assez fortement avec la densité d'électrodéposition. Avec des poutres E.L. fixées sur le montage réalisé, si l'on effectue des cycles charges-décharges en faisant croître la charge maximale à chaque cycle, on arrive à plastifier localement la poutre et obtenır des déformations irréversibles $V_{\text {nn }}$ (Fig. 13a). La figure 13b représente pour tross valeurs de $J$ l'évolution de la déformation inélastique en fonction de la charge maximale, $P=f\left(V_{\mathrm{nn}}\right)$, pour trois poutres d'épaisseurs légèrement différentes. Par une méthode d'identification inverse en E.F., c'est-à-dire partant de la réponse de la structure poutre, $P=f\left(V_{\mathrm{n}}\right)$, il est possible d'ajuster une loi élasto-plastique relative à l'élément de volume et intrinsèque au matériau. On considère une loi élasto-plastique à écrouissage isotrope 
non-linéaire, à savoir ·

$$
\begin{aligned}
& \mathrm{d} \varepsilon_{\imath \jmath}^{\mathrm{T}}=\mathrm{d} \varepsilon_{\imath \jmath}^{\mathrm{e}}+\mathrm{d} \varepsilon_{\imath \jmath}^{\mathrm{p}} \\
& \mathrm{d} \varepsilon_{\imath \jmath}^{\mathrm{e}}=\frac{1+\nu}{E} \mathrm{~d} \sigma_{\imath \jmath}-\frac{\nu}{E} \delta_{\imath \jmath} \mathrm{d} \sigma_{k k} \\
& \mathrm{~d} \varepsilon_{\imath \jmath}^{\mathrm{p}}=\mathrm{d} \lambda \frac{\partial f}{\partial \sigma_{\imath \jmath}}=\frac{3}{2} \mathrm{~d} \lambda \frac{S_{\imath \jmath}}{J_{2}(\sigma)} \text { avec } \\
& f=J_{2}(\sigma)-R-R_{0}, J_{2}(\sigma)=\left(\frac{3}{2} S_{\imath \jmath} S_{\imath \jmath}\right)^{1 / 2} \text { et } S_{\imath \jmath}=\sigma_{\imath \jmath}-\left(\delta_{\imath \jmath} / 3\right) \sigma_{k k}, \\
& \mathrm{~d} R=b\left(R^{\infty}-R\right) \overline{\mathrm{d} \varepsilon} \cdot \frac{\mathrm{d} \varepsilon}{\mathrm{p}}=\left(\frac{2}{3} \mathrm{~d} \varepsilon_{\imath \jmath}^{\mathrm{p}} \mathrm{d} \varepsilon_{\imath \jmath}^{\mathrm{p}}\right)^{1 / 2} \\
& \mathrm{~d} \lambda=\overline{\mathrm{d} \varepsilon}^{\mathrm{p}}=H(f) \frac{\mathrm{d} J_{2}(\sigma)}{\mathrm{d} R / \overline{\mathrm{d}}^{\mathrm{p}}}=H(f) \frac{\mathrm{d} J_{2}(\sigma)}{b\left(R^{\infty}-R\right)} .
\end{aligned}
$$

Dans ces équations $\varepsilon_{\imath \jmath}^{\mathrm{T}}, \varepsilon_{\imath \jmath}^{\mathrm{e}}, \varepsilon_{\imath \jmath}^{\mathrm{p}}$ sont respectivement les déformatións totale, élastique et plastıque, $S_{\imath \jmath}$ les composantes du déviateur des contraintes, $J_{2}(\sigma)$ l'équivalent de la contrainte au sens de von-Mises, $R$ la variable scalaire d'écrouissage. $R_{0}$ la valeur de la limite d'élastıcité initıale, $\mathrm{d} \lambda$ le multiplıcateur plastique égal à la déformation plastique cumulée au sens de von Mises $\overline{\mathrm{d}} \varepsilon^{\mathrm{p}}$ et $H(f)$ la fonction d'Heaviside. Ce modèle simple comporte donc trois paramètres à ajuster $R_{0}, b, R^{\infty}$, les paramètres élastıques $E$ et $\nu$ étant connuṣ.

Sur les figures $13 \mathrm{c}$, b. on reporte d'une part la simulation E.F! de l'essai de la figure 13a et d'autre part les solutions numériques optimisées des courbes $P=f\left(V_{1 \mathrm{n}}\right)$. On en déduit. figure $13 \mathrm{~d}$, les courbes élasto-plastiques intrinsèques au nickel électro-déposé en fonction de la densité de courant Le paramètre $b$ ainsi que la limite d'élasticité $R_{0}$ sont peu sensibles à $J$ ( $R_{0} \cong 300 \mathrm{MPa}$ et $b=250$ ), par contre, $R^{\infty}$ est une fonction fortement décroissante de $J$, à l'exemple de la dureté $H_{\mathrm{B}}$, le matériau étant d'autant plus écrouissable que la densité de courant est faible [19] Avec ce même modèle et suivant la mêmel technique E.F nous avons ajusté la phase de chargement des essais de nano-indentation. On retrouve des valeurs de $b$, $R_{0}$ et $R^{\infty}$ assez proches de celles détermmées en flexion, d'où la cómplémentarité de ces essais [25]. Les courbes efforts-déformations obtenues avec les paramètres relatifs à l'indentation sont également reportées sur la figure 13d. À l'aide de l'ensemble des valeurs identifiées (flexion et indentation) on peut montrer qu'il existe une relation linéaire entre le paramètre d'écrouissage $\left(R^{\infty}+R_{0}\right)$ et la dureté. soit :

$$
R_{0}+R^{\infty}=0,154 H_{\mathrm{B}}
$$

En définitive, ces expériences exécutées sur des poutres E.E. et E.L. illustrent les potentialités de ce montage vis-à-vis de la caractérisation mécanıue des matérıaux pour les micro-techniques. aussi bien dans le domaine élastıque, qu'inélastique.

\section{Conclusions}

Nous avons montré qu'à l'aide d'un dispositif expérımental de flexion de poutres, il est possıble de caractériser les propriétés mécaniques des matériaux utilisés dans le domaine des microtechniques Les dimensions des poutres testées se situent à l'échellẹ des micro-systèmes réalisés dans ce domaine, de l'ordre de $100 \mu \mathrm{m}$. Un système d'imagerie optique associé à un traitement numérique d'mages permet d'accéder à la déformée complète des structures. Liensemble du dispositif a été validé à l'aide de poutres réalısées en sllicium monocristallin dont les propriétés élastiques sont parfaitement connues. Un exemple d’application sur un matériau à propriétés $a$ 
prior inconnues, le nickel electro-déposé, est présenté On quantifie son comportement élastique et mélastique en fonction de la densité du courant d'électro-déposition puis on compare les résultats à ceux obtenus par d'autres techniques (nano-indentation et R.X )

En conclusion. on dispose à présent au sein de l'IMFC, en complément des essais de tractıon, de nano-indentation et de gonflement de plaques minces [8], d'un dispositif capable de réaliser des essais de caractérisation de matériaux en faibles dimensions.

\section{Remerciements}

Les auteurs remercient l'IMFC et l'IMM pour les structures environnementales et les nombreux conseils reçus lors de l'élaboration des poutres en silicium et en nickel.

\section{Annexe A}

- Dans le cas des poutres E L., pour le calcul analytique des relations flèches-efforts, $V=f(F)$. nous prenons, suivant que les sections droites sont rectangulaires ou trapézoïdales [20], respectivement les équations .

$$
V=\frac{4 F L^{3}}{E w t^{3}} \quad \text { ou } \quad V=\frac{12 F L^{3}(a+b)}{E\left(a^{2}+b^{2}+4 a b\right) t^{3}}
$$

Dans ces relations $L$ est la longueur entre l'encastrement et le point d'application du chargement, $t$ l'épaisseur de la poutre, $w$ sa largeur lorsque sa section est rectangulaire et $a, b$ les largeurs minimale et maximale pour une section trapézoïdale. Les autres grandeurs ont leurs significations habituelles.

- Pour les poutres E.E.. dans le cas des grandes flèches, la relation flèche-chargement le long de la direction $x$ perpendiculaire à la direction du chargement est solution de l'équation différentielle (A2), du second ordre en $V(x)$ :

$$
\begin{array}{r}
E I V^{\prime \prime}(x)-\left(S+S_{0}\right) V(x)+F x=-M_{\mathrm{a}} \\
\operatorname{avec} S=\left[\int_{0}^{L / 2}\left(V_{1}^{\prime}(x)^{2}\right) \mathrm{d} x+\int_{L / 2}^{L}\left(V_{2}^{\prime}(x)^{2}\right) \mathrm{d} x\right] \frac{E A}{2 L}
\end{array}
$$

$I$ et $A$ sont respectivement le moment quadratique et la surface d'une section droite de la poutre, $L$ la demi-longueur de la poutre, $S$ la force axiale selon $x$ résultant du chargement et des conditions aux limites, $S_{0}$ la force axıale due aux éventuelles contraintes résıduelles et $M_{\mathrm{a}}$ le moment de torsion. $V_{1}(x)$ et $V_{2}(x)$ sont respectivement les déplacements entre $[0, L / 2]$ et $[L / 2, L]$. Cette équation n'admet pas de solution analytıque Cependant. en posant la variable réduite,

$$
u=\frac{L}{2} \sqrt{\frac{S+S_{0}}{E I}}
$$

il est passıble d'intégrer (A2) et d'écrıre les relations explicites $V_{1,2}(x)=f(u, x)$ Une résolution numérıque, paramétrée par la variable $S$, de ces deux équations, tout en tenant compte des conditions aux limites, permet d'accéder à $E$ et $S_{0}$ en ajustant la solution numérique sur la déformée expérımentale. 


\section{Bibliographie}

[1] Recherches en microtechniques : réalités et perspectives - Collẹction du Livre Vert, IMFC et CETEHOR, Janvier 1992.

[2] Minottı P. et Bonnotte E., Un aperçu des problèmes posés par la conception des microactionneurs, Les Entretiens de la Technologie, 3ème Édition (Ṕris, 15-16 mars 1994).

[3] Hardwick D A., The mechanical properties of thin films, A review, Thın Solvd Fulms 153 (1987) 109-124.

[4] Doerner M F and Nix W.D., Stresses and deformation processes in thin films on substrates, CRC Critrcal Revnews in Solvd State and Material Scrences 14 (1988) 225-268.

[5] Read D.T. and Dally J.W., A new method for measuring the strenght and ductility of thins films, J. Mat. Res. 8 (1993) 1542-1549.

[6] Vlassak J.J. and Nix W.D., A new bulge test technıque for the determination of Young's modulus and Poisson's ratio thin films, J. Mat. Res. 7 (1992) 3242-3249.

[7] Tabata O., Kawahata K., Sugiyama S. and Igarashi I., Mechanical property measurements of thin films using load-deflection of composite rectangular membranes, Sensors and Actuators 20 (1989) 135-141.

[8] Bonnotte E., Delobelle P, Bornier L., Trolard B. et Tribillon G., Mise en oeuvre de deux méthodes interféromètriques pour la caractérisation mécanique des films minces par l'essai de gonflement. Application au cas du silicium monocristallin, J. Phys. III France 5 (1995) 953-983.

[9] Oliver W.C. and Pharr, G.M., An improved technique for determining hardness and elastıc modulus using load and displacement sensing indentation experiments, J. Mat. Res. 7 (1992) 1564-1583

[10] Paletto S. et Fantozzi G., Nanoindentation : théorie et applications, Rev. Comp. et Mat. Avancés 3 (1993) 139-160.

[11] Lober T.A., Huang J., Schmidt M.A. and Senturia S.D, Characterization of the mechanisms producing bending moments in polysilicon micro-cantilever beam by interferometric deflection measurements, Sd. State Sensor and Actuator (1988) p. 92.

[12] Ulrich R.K. and Zhao G., A new apparatus for measuring the ultimate strain of thin films, Thin Solvd Fulms 224 (1993) 63-68.

[13] Cuthrell R E., Gerstle F.P. and Mattox D.M., Measurement of residual stresses in films of unknown elastic modulus, Rev. Scr. Inst. 60 (1989) 1018-1020.

[14] Baker S.P., Jankowski A.F, Hong S. and Nix W.D., Mechanical properties of compositionally a modulated $\mathrm{Au}-\mathrm{Ni}$ thin films using indentation and microbeam deflection techniques, Mat. Res. Soc. Symp. Proc. (Éd. Doerner, Oliver, Pharr, Brotzen) 188 (1990) pp. 289-294.

[15] Mounaix P., Delobelle, P., Melique X., Bornier L and Lippens D., Micromachining and mechanical properties of GaInAs/InP microcantilevers, Second Int Conf. on Low Dimens. Struct. and Devices (Lisbon, 19-21 may, Portugal, 1997).

[16] Ballandras S., Daniau W., Basrour S., Robert D., Rocker S., Robert L., Blind P., Rouillay M., Bermède P., Megtert S., Liu Z., Labèque A., Rousseaux F , Ravet M F , and Hauden D., Microgrippers realized by LIGA techniques, à paraître dans Sensors and Actuators, 1997.

[17] Becker E.W., Ehrfeld W., Hagmann P., Maner A. and Munchmeyer D, Fabrication of microstructures with high aspect ratios and great structural heights by synchrotron radiation lithography, galvanoforming and plastic molding, Microelectr. Eng. 4 (1986) 35-56. 
[18] Ehrfeld W and Lehr H., Deep X-ray lithography for the production of three-dimensional microstructures from metals, polymers and ceramics. Radzation Phys. and Chemistry 45 (1995) 349-365.

[19] Robert L., Étude des propriétés mécaniques du nickel issu de la technologie LIGA. Réalisation d'un micro-préhenseur, Thèse Univ. Fr.-Comté (1997).

[20] Timoshenko S. and Gere J., Mechanics of materials (V. Nostrand Reinhold Company, 1972).

[21] Brantley W.A., Calculated elastic constants for stress problems associated with semiconductor devices, J. Appl. Phys. 44 (1973) 584-585.

[22] Tribillon G., Trolard B., Bonnotte E., Delobelle P. and Bornier L., Optical methods for the characterization of mechanical properties of thin slicon films, Opt. Meas. and Sensors for the Process Industries, Europt. Series, SPIE 2248 (1994) 198-290.

[23] Sergent A., Étude des propriétés mécaniques des matériaux en faibles dimensions par l'essai de flexion, Thèse Univ. Fr.-Comté (1997).

[24] Castex L., Lebrun J.L., Maeder G. and Sprauel, J.M., Détermination des contraintes résiduelles par diffraction des R.X., ENSAM, Paris, 1981.

[25] Sergent A., Poilane C., Robert L. and Delobelle P., La nano-indentation, le gonflement de film et la flexion de poutre ; trois essais complémentaires pour caractériser les propriétés mécaniques des matériaux en faibles épaisseurs. Application au cas du nickel électrodéposé. 40ème Coll. de Métall. de l'INSTN, 24-26 juin, Saclay, France, 1997. 